A Tractable Model of Indirect Asset Liquidity

Lucas Herrenbrueck

Simon Fraser University

Athanasios Geromichalos

University of California, Davis

October 14, 2015

Paper \# 15-3

Assets have "indirect liquidity" if they cannot be used as media of exchange, but can be traded to obtain a medium of exchange (money) and thereby inherit monetary properties. This essay describes a simple dynamic model of indirect asset liquidity, provides closed form solutions for real and nominal assets, and discusses properties of the solutions. Some of these are standard: assets are imperfect substitutes, asset demand curves slope down, and money is not always neutral. Other properties are more surprising: prices are flexible but appear sticky, and an increase in the supply of indirectly liquid assets can decrease welfare. Because of its simplicity, the model can be useful as a building block inside a larger model, and for teaching concepts from monetary theory

Department of Economics

One Shields Avenue

Davis, CA 95616

(530)752-0741

http://wp.econ.ucdavis.edu/ 


\title{
A Tractable Model of Indirect Asset Liquidity
}

\author{
September 2015 \\ Lucas Herrenbrueck, Simon Fraser University \\ Athanasios Geromichalos, University of California - Davis
}

JEL Classification: E41, E51, E52, G12

\begin{abstract}
Assets have "indirect liquidity" if they cannot be used as media of exchange, but can be traded to obtain a medium of exchange (money) and thereby inherit monetary properties. This essay describes a simple dynamic model of indirect asset liquidity, provides closed form solutions for real and nominal assets, and discusses properties of the solutions. Some of these are standard: assets are imperfect substitutes, asset demand curves slope down, and money is not always neutral. Other properties are more surprising: prices are flexible but appear sticky, and an increase in the supply of indirectly liquid assets can decrease welfare. Because of its simplicity, the model can be useful as a building block inside a larger model, and for teaching concepts from monetary theory.
\end{abstract}

Keywords: monetary-search models, asset liquidity, asset prices, monetary policy

Recently, interest has increased in tractable models of asset liquidity that are easy to analyze and can provide benchmarks for understanding the relationship between monetary policy and asset market frictions. For example, Williamson (2012), Andolfatto and Williamson (2015), and Rocheteau, Wright, and Xiao (2014) analyze policy using models in which multiple assets can take the role of a medium of exchange, usually to different degrees, which makes them imperfect substitutes and the question of their relative supplies interesting. Furthermore, a number of recent papers have suggested that the notion of asset liquidity may be the key to rationalizing some long-standing asset pricing-related puzzles (see for example Lagos, 2010, Geromichalos, Herrenbrueck, and Salyer, 2013, and Jung and Lee, 2015). 
The assumption that assets such as US Treasuries or claims to capital serve as media of exchange is popular in monetary theory (going back to Geromichalos, Licari, and Suarez-Lledo, 2007, and Lagos and Rocheteau, 2008), but is sometimes questioned based on realism. In Geromichalos and Herrenbrueck (2012), we demonstrated that indirect asset liquidity can support many of the same conclusions, while offering an alternative microfoundation of imperfect asset substitutability: in this conception of liquidity, assets are substitutes to money because agents can sell them in a secondary financial market, and thus obtain the money needed to make a purchase. This detail is important because it implies that the structure of financial markets has first-order consequences for the 'moneyness' of different types of assets, and therefore their trading volumes and prices.

However, the full version of our model did not admit a closed-form description of the solution. The goal of this essay is to simplify the model in a way which preserves its essential features, but increases its usefulness as a building block for general applications. In addition, we extend the model to the case of nominal bonds. The new model sheds light on some latent results that were not highlighted in the earlier paper: most notably, we show that in contrast to conventional wisdom, an increase in the supply of liquid assets can lead to a decrease in welfare.

\section{The model}

The model described here is a simplified version of Geromichalos and Herrenbrueck (2012), and we limit the exposition to the essential details. As in the framework of Lagos and Wright (2005), time is discrete and goes on forever. At the end of each period, a competitive "centralized" market opens in which agents produce and consume a general consumption good, and allocate their wealth between two different assets.

The first asset, "money" $M$, is liquid in the sense that it can be used to purchase special consumption goods. These goods are traded in markets subject to search frictions, where agents are anonymous and lack commitment. Therefore, a medium of exchange is required, and money is the only asset that can play this role. The second asset is a real discount bond $b$ which is illiquid in the sense that it cannot be used to purchase consumption. Instead, these bonds can be liquidated in an over-the-counter asset market (OTC henceforth) for money; because agents 
anticipate this, they may value the bonds at a liquidity premium in the centralized market even though the bonds are never used as media of exchange. In this sense, bond liquidity is indirect.

There are two distinct types of agents (as in Rocheteau and Wright, 2005). Producers of special goods are trivial in this model: they can never consume special goods and therefore have no demand for liquid assets. All other agents (whom we simply refer to as "agents" from here on) value consumption of $q$ units of the special consumption good at $u(q)$, a strictly increasing and concave function. The cost of production is linear with slope normalized to 1 , so $u(q)-q$ denotes the surplus in every trade for the special good. There is a quantity $q^{*}$ which satisfies $u^{\prime}\left(q^{*}\right)=1$; we call it the "first-best" quantity because it maximizes the joint surplus of consumer and producer. Because a medium of exchange is required, however, actual trade in the special good will often be for less than this quantity.

Agents discount the future at rate $\beta<1$. The money supply grows at rate $\mu>\beta-1$, which determines inflation in steady state; but for convenience, we summarize this information with the parameter $i \equiv(1+\mu-\beta) / \beta .{ }^{1}$ Newly printed money is introduced via lump-sum transfers to agents in the centralized market. ${ }^{2}$

At the beginning of every period, a proportion $\ell<1$ of agents learn that they have the opportunity to consume the special good in this period. However, because at the end of the last period they did not know if the consumption opportunity would arise today, all agents hold the same amounts of money and bonds; in particular, some hold money that they do not need in the same period, and others hold bonds that they would like to sell in order to get more money.

This portfolio reallocation happens in an OTC market characterized by frictions. $^{3,4}$ The first friction is that trade is bilateral and matching is random, and the total number of matches is $f<\min \{\ell, 1-\ell\}$ so that nobody matches with certainty. Second, in the full model, we modeled the terms of trade using proportional bar-

${ }^{1}$ This parameter can be interpreted as the cost of liquidity. It is sometimes called the nominal interest rate; in a world where liquidity premia exist, it should be clarified that $i$ would be the nominal interest rate on an asset that is either perfectly illiquid or in such large supply so that its liquidity properties do not matter at the margin.

${ }^{2}$ Whether monetary policy is set in terms of inflation or in terms of the fiscal transfer matters a great deal, especially in a liquidity trap (Herrenbrueck, 2014; Andolfatto and Williamson, 2015).

${ }^{3}$ Other models of portfolio reallocation include Alvarez, Atkeson, and Kehoe (2002), Berentsen, Camera, and Waller (2007), Berentsen, Huber, and Marchesiani (2014), and Lagos and Zhang (2014).

${ }^{4}$ OTC market structure is empirically relevant for a large class of assets, including some that we think of as very liquid (Duffie, Gârleanu, and Pedersen, 2005; Afonso and Lagos, 2014). 
gaining; here, to make things tractable, we assume that the asset seller makes a take-it-or-leave-it offer and therefore extracts the full surplus from the OTC trade. This simplification matters for welfare and for the OTC market price, but all other results are qualitatively unchanged. It also eliminates an asset pricing indeterminacy which can otherwise arise for particular parameters.

An equilibrium will be characterized by three endogenous variables. The first is the quantity of special goods bought by consumers who did not obtain additional money in the OTC market, denoted by $q_{0}$. The second is the quantity of special goods bought by consumers who did obtain additional money in the OTC market, denoted by $q_{1}>q_{0}$. The third equilibrium object is the real price of bonds in the centralized market, denoted by $\psi$.

To make things even simpler, we want to restrict attention to equilibria in which asset buyers are never constrained by their money holdings in the OTC. A sufficient condition which assures this is that inflation is not too large, in particular: $i<(\ell-f)\left[u^{\prime}\left(q^{*} / 2\right)-1\right]$. We will maintain this assumption from now on.

We might also be interested in characterizing welfare, which is here given by the surplus of consuming special goods, averaged between consumers of special goods who had the opportunity to rebalance and those who did not:

$$
\mathcal{W}=(\ell-f)\left[u\left(q_{0}\right)-q_{0}\right]+f\left[u\left(q_{1}\right)-q_{1}\right]
$$

\subsection{Equilibrium with real bonds}

We can now state three equations determining general equilibrium in the endogenous variables $\left\{q_{0}, q_{1}, \psi\right\}$.

(Money demand)

$$
\begin{aligned}
i & =(\ell-f)\left[u^{\prime}\left(q_{0}\right)-1\right]+f\left[u^{\prime}\left(q_{1}\right)-1\right] \\
q_{1} & =\min \left\{q^{*}, q_{0}+b\right\} \\
\psi & =\beta\left[1+f\left(u^{\prime}\left(q_{1}\right)-1\right)\right]
\end{aligned}
$$$$
\text { (OTC trade) }
$$

(Bond demand)

This formula for the bond price $\psi$ can be interpreted as the product of the "fundamental value" $\beta$ and a liquidity premium. (In particular, it could easily imply a negative real interest rate if $i$ is large enough.) Comparative statics with respect to bond supply or bond liquidity are now straightforward. First, observe that if $b \geq q^{*}-\left(u^{\prime}\right)^{-1}[1+i /(\ell-f)]$, then an OTC bond seller will not sell all of her bonds. In this case, bond supply is "abundant", and it does not affect the equilibrium 
quantities, prices, or welfare at the margin. But bond liquidity does matter, even if the bond supply is abundant: an increase in $f$ will make it easier for agents to obtain cash ex post, and they will therefore demand less ex ante. This will drive down real balances (which may matter on its own, as an increase in the price level), and for a large enough increase in $f$, real balances will be so low that bonds are not abundant after all.

This other case, where an OTC bond seller will sell all of her bonds and bond supply is therefore "scarce", is obtained when $b<q^{*}-\left(u^{\prime}\right)^{-1}[1+i /(\ell-f)]$. It is much more interesting: increases in bond supply $b$ reduce real balances pre-trade $\left(q_{0}\right)$ but increase them post-trade $\left(q_{1}\right)$, and therefore also increase the bond yield $1 / \psi-1$; the aggregate bond demand curve is downward sloping.

In the scarce case, the effect of the bond supply on welfare is more complex and depends on the shape of the utility function:

$$
\frac{d \mathcal{W}}{d b}=f(\ell-f) \frac{\left[u^{\prime}\left(q_{1}\right)-1\right] \cdot u^{\prime \prime}\left(q_{0}\right)-\left[u^{\prime}\left(q_{0}\right)-1\right] \cdot u^{\prime \prime}\left(q_{1}\right)}{(\ell-f) u^{\prime \prime}\left(q_{0}\right)+f u^{\prime \prime}\left(q_{1}\right)}
$$

In general, the sign of the derivative is ambiguous, but we can say more in two special cases. First, if the bond supply approaches the abundant level, we have $q_{1} \rightarrow q^{*}$ (by definition of "abundant") and therefore $u^{\prime}\left(q_{1}\right) \rightarrow 1$. As long as the reasonable assumption $u^{\prime \prime}\left(q^{*}\right)<0$ is satisfied, this implies that $d \mathcal{W} / d b$ is always negative when $b$ approaches the abundant level. Second, because $u$ is concave, $u^{\prime}\left(q_{0}\right)$ is always greater than $u^{\prime}\left(q_{1}\right)$. If $u^{\prime \prime}\left(q_{1}\right)$ is greater in absolute value than $u^{\prime \prime}\left(q_{0}\right)$ (both are of course negative, so $u^{\prime \prime \prime}(q) \leq 0$ would be a sufficient condition for that), then welfare is always decreasing in the real supply of bonds - an outcome in contrast to most other models of bond liquidity.

We now turn our attention to a quadratic utility function for which we can compute general equilibrium explicitly. Let $u(q) \equiv(1+\sigma) q-\frac{1}{2} q^{2}$, which has the properties $u^{\prime}(q)-1=\sigma-q$ and $q^{*}=\sigma$. First, if $b \geq i /(\ell-f)$, then bond supply is abundant and does not affect the equilibrium:

$$
\begin{aligned}
& q_{0}=\sigma-\frac{i}{\ell-f} \\
& q_{1}=\sigma \\
& \psi=\beta
\end{aligned}
$$


If, on the other hand, $b<i /(\ell-f)$, then the bond supply is scarce and the equilibrium is:

$$
\begin{aligned}
& q_{0}=\sigma-\frac{i}{\ell}-\frac{f}{\ell} b \\
& q_{1}=\sigma-\frac{i}{\ell}+\frac{\ell-f}{\ell} b \\
& \psi=\beta\left[1+\frac{f}{\ell}(i-(\ell-f) b)\right]
\end{aligned}
$$

This formula for the bond price $\psi$ has some very convenient properties. The liquidity premium is linear in the cost of carrying money $(i)$ and in the aggregate supply of bonds $(b)$. It is also strictly increasing (although not linear) in the probability of trade in the OTC $(f)$ for the entire range $b \in[0, i /(\ell-f))$ where the liquidity premium is positive, and this range is itself expanding in $i$ and $f$.

Perhaps surprisingly, the bond price is independent of $\sigma$ (which can be interpreted as the size of the liquidity shocks, just as $\ell$ describes their frequency). The intuition is that the liquidity premium measures the ability of the asset to catalyze a need to obtain liquidity ex post, and this need arises from the fact that liquidity is costly and therefore agents hold too little of it ex ante. If $\sigma$ increases, liquidity becomes more valuable both ex post and ex ante, so that the need for rebalancing is exactly unchanged in equilibrium. This independence result is specific to the quadratic utility function, however, and the fact that $\sigma$ only affects its first derivative but not the second.

As the quadratic utility function satisfies $u^{\prime \prime \prime}(q)=0$, it always implies the counterintuitive result that welfare is decreasing in the real supply of bonds. Using $u^{\prime \prime}(q)=-1$, we can compute directly:

$$
\frac{d \mathcal{W}}{d b}=-\frac{f(\ell-f)}{\ell} b
$$

\subsection{Equilibrium with nominal bonds}

Instead of a real bond in supply $b$, say that there is a nominal bond in supply $B$. In this case, the real bond price is not very meaningful, so we want to use the nominal bond price $p$ instead. (Using the money demand equation, it is easy to show that $p \leq 1$, i.e. the nominal yield on this bond cannot be negative even though the real one can.) The equations governing general equilibrium are as follows: 
(Money demand)

$$
\begin{aligned}
i & =(\ell-f)\left[u^{\prime}\left(q_{0}\right)-1\right]+f\left[u^{\prime}\left(q_{1}\right)-1\right] \\
q_{1} & =\min \left\{q^{*}, q_{0}\left(1+\frac{B}{M}\right)\right\} \\
p & =\frac{1}{1+i}\left[1+f\left(u^{\prime}\left(q_{1}\right)-1\right)\right]
\end{aligned}
$$$$
\text { (OTC trade) }
$$

(Bond demand)

There are two differences from the previous model. A technical difference is that the equation describing how the post-trade quantity $q_{1}$ is determined from the pre-trade quantity $q_{0}$ is now multiplicative instead of additive. This may seem trivial, but it makes it possible to use a CRRA function for $u(q)$ in order to get closed-form solutions, in addition to the quadratic one. The substantive difference is that money is no longer neutral, at least in the case where bonds are scarce.

We will discuss the neutrality of money in our model in Section 2. But first, we continue our quest for closed-form solutions and use the quadratic utility function from Section 1.1 again. If $B / M \geq i /[(\ell-f) \sigma-i]$, then the bond supply is abundant and the equilibrium is:

$$
\begin{aligned}
q_{0} & =\sigma-\frac{i}{\ell-f} \\
q_{1} & =\sigma \\
p & =\frac{1}{1+i}
\end{aligned}
$$

If, on the other hand, $B / M<i /[(\ell-f) \sigma-i]$, then the bond supply is scarce and the equilibrium is:

$$
\begin{aligned}
q_{0} & =\frac{M}{\ell M+f B}(\ell \sigma-i) \\
q_{1} & =\frac{M+B}{\ell M+f B}(\ell \sigma-i) \\
p & =\frac{1}{1+i}\left(1+f \frac{i(M+B)-(\ell-f) \sigma B}{\ell M+f B}\right)
\end{aligned}
$$

The resulting equations are more complex than Equations (2); for example, the bond price is no longer linear in the bond supply. However, we also gain some new insights. For one, the bond price is now decreasing in $\sigma$, the size of the liquidity shocks. Intuitively, an increase in the overall demand for liquidity increases the 
demand for money (which is weighted by $\ell$ ) by more than the demand for bonds (which is weighted by $f<\ell$ ), which drives down the bond price and increases interest rates on that bond, potentially to a point where bonds are no longer scarce and their price is at the fundamental level.

As suggested earlier, we can also get closed form solutions for the model with nominal bonds if we use a CRRA utility function, which we could not do in the model with real bonds. Let $u(q)=q^{1-\gamma} \sigma^{\gamma} /(1-\gamma)$, with $\gamma>0$, which again has the property that $q^{*}=\sigma$. Bonds are abundant if $B / M \geq[(i+\ell-f) /(\ell-f)]^{1 / \gamma}-1$, and in this case the equilibrium is:

$$
\begin{aligned}
q_{0} & =\sigma\left(\frac{\ell-f}{i+\ell-f}\right)^{1 / \gamma} \\
q_{1} & =\sigma \\
p & =\frac{1}{1+i}
\end{aligned}
$$

If, on the other hand, $B / M<[(i+\ell-f) /(\ell-f)]^{1 / \gamma}-1$, then the bond supply is scarce and the equilibrium is:

$$
\begin{aligned}
q_{0} & =\sigma\left(\frac{\ell-f+(1+B / M)^{-\gamma} f}{i+\ell}\right)^{1 / \gamma} \\
q_{1} & =\sigma\left(\frac{(\ell-f)(1+B / M)^{\gamma}+f}{i+\ell}\right)^{1 / \gamma} \\
p & =\frac{1}{1+i}\left(1-f+f \frac{i+\ell}{(\ell-f)(1+B / M)^{\gamma}+f}\right)
\end{aligned}
$$

As in Equation (4c), the bond price is not linear in any variable, but depending on the question, it may have other attractive properties. Note that it is independent of the size of the liquidity shock $\sigma$, as was the case in Equation (2c). The deep reason is the same: if liquidity becomes more valuable then the demand for liquid bonds goes up, but this is exactly offset by the endogenous change in the price level that makes their effective supply go down. Or in other words, any increase in the value of liquidity falls proportionally on money and bonds, unlike in Equations (4). This result makes clear that how exactly we model the "demand for liquidity" matters in subtle ways. 


\section{Discussion}

In this essay, we have constructed a simple model of indirect asset liquidity that admits convenient closed-form solutions for a variety of applications. In a model with real bonds, the resulting equilibrium equations are exceptionally simple: for example, the price of real bonds is linear in the bond supply and in the cost of liquidity. In a model with nominal bonds, the equations become less clean, but clearly illustrate the non-neutrality of money. To analyze this point in more detail, consider the determination of the general price level, defined as the ratio of money supply to real money demand, $P \equiv M / q_{0}$. Assuming that the bonds are scarce in OTC trade, we can transform Equation (4a) from the quadratic model to obtain:

$$
P=\frac{\ell M+f B}{\ell \sigma-i}
$$

Alternatively, and still assuming that the bonds are scarce enough, we can use the CRRA model and Equation (6a) to obtain:

$$
P=\frac{(i+\ell)^{1 / \gamma}}{\sigma}\left[(\ell-f) M^{-\gamma}+f(M+B)^{-\gamma}\right]^{-1 / \gamma}
$$

As the equations reveal, the price level is increasing as a function of inflation, decreasing as a function of the size or frequency of the liquidity shocks, and increasing in the liquidity of asset markets. Most importantly, of course, money is not neutral. Why? The reason is that the price level is proportional to the total stock of nominal assets that have a liquidity role, not to the money supply alone. When only one of these assets increases in quantity, then the price level will not increase in proportion. Changing the money supply via open market operations will only amplify the non-neutrality (see also Rocheteau, Wright, and Xiao, 2014): an increase in $M$ financed by a decrease in $B$ will increase the bond price (i.e. reduce nominal interest rates on the bond), and will not change the overall price level as much as a helicopter drop would have, even though prices are perfectly flexible in this model. Prices will look sticky.

In contrast to many of the models listed at the beginning of this essay, the simple model described here is not capable of exhibiting a liquidity trap situation where bond demand becomes flat at low interest rates. However, this is not true of the concept of indirect liquidity in general: Herrenbrueck (2014) describes 
a continuous-time model of indirect liquidity in which a liquidity trap can exist.

The idea that many assets contribute to providing monetary services is also the foundation of the Divisia monetary aggregates originally proposed by Barnett $(1978,1980)$. Their construction involves first determining what the fundamental cost of liquidity is ( $i$ in this model), then weighting assets according to their "user cost". For example, if it happens that $i=6 \%$, then an asset yielding $3 \%$ receives half the weight of cash (which yields $0 \%$ ) in the monetary aggregate. Our model identifies a problem with this method. In Equations (7) and (8), the weights on money and bonds can be interpreted as their average contributions to the supply of liquidity, while the distance between their yields and the fundamental yield $i$ measures their marginal contributions. For example, an increase in bond liquidity will increase the overall liquidity supply and reduce the bond yield, so the Divisia method would get the direction right; but an increase in the bond supply will increase the bond yield, leading the Divisia method to underestimate the increase in the overall liquidity supply. ${ }^{5}$

Despite its resemblance to the undergraduate textbook model of money demand, this model has more subtle implications. For one, while the demand for liquid assets certainly depends on the total demand for goods, it is not necessarily true that different levels of income are "associated" with different interest rates, even for a fixed combination of asset supplies. As the solutions above make clear, bond yields may depend on the overall demand for liquidity, but not necessarily - because a change in this demand may be distributed on all assets in proportion to their existing uses, or it may not be. The second difference from the simple textbook model is that changes in the interest rate (real or nominal) on a particular liquid asset do not reveal much about the interest rate available to anyone not issuing this asset, or even about $i$, a point which has of course been recognized for a long time. ${ }^{6}$ However, questions of this kind are exactly what our simple model of asset liquidity can usefully address. ${ }^{7}$

\footnotetext{
${ }^{5} \mathrm{Li}$, Rocheteau, and Weill (2012) also study the weights of partially liquid assets in a monetary aggregate, and emphasize the distinction between the private value and the social value of an asset.

${ }^{6}$ An important application of this argument is that the effect of open market purchases of longterm government bonds on the prices and quantities of other assets, such as physical capital, is not obvious when these assets differ in liquidity (Herrenbrueck, 2014).

${ }^{7}$ For example, Geromichalos and Herrenbrueck (2015) use it to study the strategic interaction of asset issuers, when each of their assets can have this indirect liquidity role.
} 


\section{References}

Afonso, G. and R. Lagos (2014). Trade dynamics in the market for federal funds. Technical report, National Bureau of Economic Research.

Alvarez, F., A. Atkeson, and P. J. Kehoe (2002, February). Money, interest rates, and exchange rates with endogenously segmented markets. Journal of Political Economy 110(1), 73-112.

Andolfatto, D. and S. Williamson (2015). Scarcity of safe assets, inflation, and the policy trap. Journal of Monetary Economics.

Barnett, W. A. (1978). The user cost of money. Economics Letters 1(2), 145-149.

Barnett, W. A. (1980). Economic monetary aggregates. an application of index number and aggregation theory. Journal of Econometrics 14(1), 11-48.

Berentsen, A., G. Camera, and C. Waller (2007). Money, credit and banking. Journal of Economic Theory 135(1), 171-195.

Berentsen, A., S. Huber, and A. Marchesiani (2014). Degreasing the wheels of finance. International Economic Review 55(3), 735-763.

Duffie, D., N. Gârleanu, and L. H. Pedersen (2005, November). Over-the-counter markets. Econometrica 73(6), 1815-1847.

Geromichalos, A. and L. Herrenbrueck (2012). Monetary policy, asset prices, and liquidity in over-the-counter markets. Working paper, University of California, Davis.

Geromichalos, A. and L. Herrenbrueck (2015). The strategic determination of the supply of liquid assets. mimeo.

Geromichalos, A., L. Herrenbrueck, and K. Salyer (2013). A search-theoretic model of the term premium. Working paper, University of California, Davis.

Geromichalos, A., J. M. Licari, and J. Suarez-Lledo (2007, October). Monetary policy and asset prices. Review of Economic Dynamics 10(4), 761-779. 
Herrenbrueck, L. (2014). Quantitative easing and the liquidity channel of monetary policy. Working paper, Simon Fraser University.

Jung, K. M. and S. Lee (2015). A liquidity-based resolution of the uncovered interest parity puzzle. Working paper.

Lagos, R. (2010, November). Asset prices and liquidity in an exchange economy. Journal of Monetary Economics 57(8), 913-930.

Lagos, R. and G. Rocheteau (2008, September). Money and capital as competing media of exchange. Journal of Economic Theory 142(1), 247-258.

Lagos, R. and R. Wright (2005, June). A unified framework for monetary theory and policy analysis. Journal of Political Economy 113(3), 463-484.

Lagos, R. and S. Zhang (2014). Monetary exchange in over-the-counter markets: A theory of speculative bubbles, the fed model, and self-fulfilling liquidity crises.

Li, Y., G. Rocheteau, and P.-O. Weill (2012). Liquidity and the threat of fraudulent assets. Journal of Political Economy 120(5), 815-846.

Rocheteau, G. and R. Wright (2005). Money in search equilibrium, in competitive equilibrium, and in competitive search equilibrium. Econometrica 73(1), 175-202.

Rocheteau, G., R. Wright, and S. X. Xiao (2014). Open market operations. mimeo.

Williamson, S. D. (2012). Liquidity, monetary policy, and the financial crisis: A new monetarist approach. The American Economic Review 102(6), 2570-2605. 